\title{
Angiotensin-converting enzyme 2 SNPs as the common genetic loci and optimal early identification genetic markers for COVID-19
}

\section{Heng Zou}

Fudan University Affiliated Public Health Clinical Center: Shanghai Public Health Clinical Center Qiuyue Li

Fudan University Affiliated Public Health Clinical Center: Shanghai Public Health Clinical Center Jun Chen

Fudan University Affiliated Public Health Clinical Center: Shanghai Public Health Clinical Center

Songmei Liu

Wuhan University Zhongnan Hospital

Shanshan Liu

Fudan University Affiliated Public Health Clinical Center: Shanghai Public Health Clinical Center

\section{Yan Ma}

Fudan University Affiliated Public Health Clinical Center: Shanghai Public Health Clinical Center

\section{Xiaomeng He}

Fudan University Affiliated Public Health Clinical Center: Shanghai Public Health Clinical Center

\section{Yun Ling}

Fudan University Affiliated Public Health Clinical Center: Shanghai Public Health Clinical Center Jianghua Zheng

shang hai shi pu dong xin qu zhou pu yi yuan: Shanghai Pudong New District Zhoupu Hospital

Christopher Corpe

King's College London

\section{Hongzhou Lu}

Fudan University Affiliated Public Health Clinical Center: Shanghai Public Health Clinical Center Jin Wang ( $\nabla$ wjincityu@yahoo.com )

Fudan University https://orcid.org/0000-0002-0062-2489

\section{Research Article}

Keywords: ACE2, SNP, COVID-19, cardiovascular risks

Posted Date: November 1st, 2021

DOl: https://doi.org/10.21203/rs.3.rs-1018735/v1 
License: (c) (i) This work is licensed under a Creative Commons Attribution 4.0 International License. Read Full License 


\section{Abstract \\ Background}

Angiotensin-converting enzyme 2 (ACE2) is implicated as a host cell receptor that causes infection in the pathogenesis of Coronavirus disease 2019 (COVID-19), and its genetic polymorphisms in the ACE2 gene may promote cardiovascular disease and systemic inflammatory injury in COVID-19. Hence, genetic background may potentially explain the broad inter-individual variation of disease susceptibility and/or severity.

\section{Methods}

The genetic susceptibility to COVID-19 by examining single-nucleotide polymorphisms (SNPs) of ACE2 was analyzed in 196 patients with COVID-19 and 210 normal controls using TaqMan genotyping assay.

\section{Results}

We demonstrated that ACE2 SNP rs4646142, rs6632677, and rs2074192 were associated with COVID-19 (all $P<0.05$ ), and the differences of ACE2 SNPs rs4646142 and rs6632677 were correlated with COVID19 related systemic inflammatory injury and cardiovascular risk. Specially, rs4646142 was associated with high-sensitive C-reactive protein (hs-CRP), prealbumin (PAB), apolipoprotein A (APOA), high-density lipoprotein (HDL), and acid glycoprotein (AGP). Rs6632677 was also associated with elevated CRP and haptoglobin (HPT).

\section{Conclusions}

Our results suggest that early identification of these individuals can provide a possible strategy for preventing the spread of the COVID-19, and ACE2 SNPs rs4646142 and rs6632677 may be a common genetic loci and optimal early identification genetic marker for COVID-19 with cardiovascular risks.

\section{Background}

Severe acute respiratory syndrome coronavirus 2 (SARS-CoV-2), the virus that causes Coronavirus disease 2019 (COVID-19) via respiratory infection, was first discovered in Wuhan, China, in December 2019. Since then, the virus has become deeply entrenched in many countries and territories, and is an enormous threat to global public health and the economy. As of June 02,2021 , over 170 million cases of COVID-19 have been confirmed worldwide and 3.56 million deaths have resulted from SARS-CoV-2 infection (1). The most common clinical symptoms of COVID-19 are fever (2), dry cough, headache (3), sore throat, nausea, breathlessness, loss of taste or smell (4) while some remain asymptomatic $(5,6)$. Morbidity and mortality caused by COVID-19 are also closely associated with past co-existing diseases, 
especially cardiovascular diseases(7). Meanwhile, human genetic factors may lead to the increased transmissibility of SARS-CoV-2 and contribute to persistent progressive disease observed in a part of infected people, but these genetic factors are largely unknown (8). Firstly, four mathematical models were constructed to predict SARS-CoV-2 transmission and the effectiveness of eradication strategies (9). The effective detection rate and detection time of SARS-CoV-2 real-time reverse transcriptase-polymerase chain reaction (rRT-PCR) analysis with the sensitivity and specificity of various antibody detection methods were reviewed (10). We have further investigated that the determinants of disease severity seem to be mainly derived from host factors, such as age and lymphopenia, while genetic variation of the virus has no significant effect on the outcomes of COVID-19 (11). Furthermore, abundant studies have indicated the possible associations of ACE2 single-nucleotide polymorphisms (SNPs) and cardiovascular risks in patients with cardiovascular disease (12-14). These observations suggest how much of the variation in COVID-19 disease severity may be explained by genetic types. Hence, early warning of inflammatory infections and cardiovascular disease risks by identifying host genetic DNA polymorphisms (variants), including excessive immune responses to viruses, will greatly promote the development of new prevention and/or treatment strategies for COVID-19.

At present, the cognition of the physicochemical property of SARS-CoV-2 mostly derived from research on SARS-CoV and MERS-CoV. As is well-known, angiotensin-converting enzyme 2 (ACE2) was highly expressed in human pneumocytes, intestinal epithelial cells and endothelial cells $(15,16)$. Given the importance of 3D structure of proteins in protein-protein interaction (PPI) it's not surprising that any process resulting in ACE2s' 3D structure change could influence COVID-19s' cell entry. Accumulating evidence has shown that 3D structure of ACE2 might be influenced at both transcriptional and posttranscriptional levels. SNPs are able to impact on protein function, structure, stability, and abundance (17). Therefore, the fundamental role of ACE2 in virus infection created this hypothesis that different complications to SARS-CoV-2 might be due to different ACE2 SNPs (18). Since the outbreak of the COVID19 pandemic several authors have speculated about the role of the ACE and ACE2 gene polymorphisms in disease susceptibility and severity. However, as to what extent the variable response to complications (inflammatory infections and cardiovascular disease risks) with COVID-19 is influenced by the variability of the hosts' genetic background was almost unclear.

Using SNPs for DNA sequence comparisons are commonly used in genetic diversity and evolutionary studies, and it is especially helpful in identifying the mutated coronavirus genomes (19). Recently, ACE2 polymorphisms were mentioned in human population, and ACE2 expression could impact the susceptibility of people to cardiovascular, hypertension, dyslipidemia, diabetes and SARS-CoV-2 infection (20). For example, 8 ACE2 SNPs (rs2074192, rs233575, rs4240157, rs4646156, rs4646188, rs1978124, rs2048683, and rs879922) were related to Type 2 diabetes mellitus (T2D) (13). Furtherly, these ACE2 SNPs were correlated with diabetic related cardiovascular complications (13). The association of ACE2 (rs233575) gene polymorphisms with blood pressure was also found in adolescents (21). More discrepant outcomes to COVID-19 disease in connected with ACE2 polymorphisms (rs2074192) in obese, smoking males (21). Besides, two SNPs (K26R and S331F), observed missense variants, can reduce ACE2 receptor affinity for the spike (S) protein (22). 
In this study, we speculated that genetic factors in the ACE2 gene are likely to impact the susceptibility to COVID-19 with previous coexisting disease. By establishing the SNP genotyping method and collecting literature to ACE2 polymorphisms, we chose rs2074192, rs6632677, rs4646142, rs2048683, rs4240157, five intronic SNP in ACE2, based on different genotyping, the analysis of clinical indicators is performed in COVID-19 patients. We verified the pathological degree of the COVID-19 was associated with hypertension and diabetes. Furtherly, our observations illustrated that ACE2 SNPs rs4646142 and rs6632677 may be an optimal genetic susceptibility marker for COVID-19 related cardiovascular complications. In summary, this study aims to present possible variants in the regulatory regions of ACE2, which may lead to marked inflammatory infections and cardiovascular disease clinical indicators variations in the prevalence and mortality of COVID-19. These may be further used in the genetic association study of patients with SARS-CoV-2 infection.

\section{Methods}

\subsection{Clinical subjects and specimens}

A total of 196 whole blood samples were collected from COVID-19 patients who were confirmed to have COVID-19 by qRT-PCR (Quantitative Real-time PCR) from the affiliated hospital Shanghai Public Health Clinical Center of Fudan University (Shanghai, China). A total of 210 whole blood samples were collected from normal subjects in the affiliated hospital Shanghai Public Health Clinical Center of Fudan University. The demographic and baseline clinical characteristics included smoking, drinking, allergy, surgery, previous coexisting disease. All procedures were reviewed and approved by the Ethics Committee of Shanghai Public Health Clinical Center, Fudan University, and have obtained written informed consents from all subjects.

\subsection{Genomic DNA extraction}

Peripheral blood $(2 \mathrm{~mL})$ was obtained from COVID-19 patients, and was stored in ethylenediaminetetraacetic acid tubes at $-20^{\circ} \mathrm{C}$ until required. Genomic DNA (gDNA) was extracted from the peripheral blood samples using a TIANamp Blood DNA Kit (TIANGEN Biotech, Cat"\#. DP304-02, Beijing, China) according to the manufacturer's protocol. Finally, gDNA was dissolved in $70 \mu \mathrm{L}$ RNA-Free water. gDNA integrity and concentration were assessed about 20-50 ng/ $\mu \mathrm{L}$ using Nano Drop ND-2000 (ThermoFisher, Waltham, MA).

\subsection{Genotyping assay}

Five ACE2 SNPs (rs4646142, rs2048683, rs4240157, rs6632677, rs2074192) (ThermoFisher, Cat": 4351379, Waltham, MA) were selected based on summarizing the literature and HapMap database. ACE2 genotyping was performed by TaqMan fluorescence probe assay (ThermoFisher, Waltham, MA). In total, $20 \mathrm{ng}$ of gDNA per sample was used for reaction. We performed PCR in a total reaction volume of $5 \mu \mathrm{L}$, including $2.5 \mu \mathrm{L}$ of $2 \times$ Master Mix (ThermoFisher, Cat": 4440038 , Waltham, MA), $0.25 \mu \mathrm{L}$ of $20 \times$ TaqMan® Assays Buffer (ThermoFisher, Cat": 4351379, Waltham, MA), 10 ng of gDNA, and dd $_{2} \mathrm{O}$. The 
reaction was initiated at $95^{\circ} \mathrm{C}$ for 10 min followed by $95^{\circ} \mathrm{C}(15 \mathrm{~s})$ and $60^{\circ} \mathrm{C}(1 \mathrm{~min})$ for 40 cycles by using the LightCycler 480 II Instrument (Roche Molecular Systems, Inc).

\subsection{Statistical analysis}

Allele frequencies were calculated following the genotypes. categorical variables were presented as number (\%) and continuous variables were presented as mean \pm standard deviation (SD). Categorical variables were compared using Chi-square-test and continuous variables were compared using one-way analysis of variance. The Odds ratio (OR) between control genotype and SARS-CoV-2 risk genotype for each ACE2 SNP among categorical variables was evaluated using binary logistic regression. a 95\% confidence interval. A $P$ value less than 0.05 was considered statistically significant. All probabilities are two-tailed.

\section{Results}

\subsection{Characteristics of the study participants}

Among 196 patients who had a laboratory-firmed SARS-CoV-2 infection, there were two cases of mild type, 184 cases of common, three cases of critical disease, and seven cases of severe COVID-19 (Table 1), which were classified according to the latest WHO regulations (23). The correlation between the COVID-19 severity degree and baseline clinical characteristics were showed in Table 1. We found that the proportion of older patients ( $\geq 60$ years old) with critical and severe COVID-19 were higher than common $(P=0.029)$. The prevalence of major coexisting illnesses such as heart disease $(P=0.021)$ and Pulmonary disease $(P<0.001)$ were also more likely to exist differently in these patients with COVID-19. Interestingly, lower percentage of patients with drug allergy were discovered in the patients with wild, critical disease and severe COVID-19. From the disease origin perspective, there was significantly different disease types of COVID-19 in the inland and oversea patient $(P<0.001)$. The most-reported symptoms of COVID-19 were fever (84.2\%), dry cough (48.9\%) and are shown in Table 2. Furthermore, the most common initial therapies were oxygen and arbidol antiviral treatment (Table 2). 
Table 1

The baseline clinical characteristics of the study population.

\begin{tabular}{|c|c|c|c|c|c|}
\hline Characteristics & $\begin{array}{l}\text { Mild } \\
(n=2)\end{array}$ & $\begin{array}{l}\text { Common } \\
(n=184)\end{array}$ & $\begin{array}{l}\text { Severe } \\
(n=7)\end{array}$ & $\begin{array}{l}\text { Critical } \\
(n=3)\end{array}$ & $P$ value \\
\hline Age, mean \pm SD & $18.50 \pm 3.536$ & $49.01 \pm 14.36$ & $60.14 \pm 15.19$ & $66.33 \pm 7.371$ & $<0.001$ \\
\hline Distribution - $\mathrm{n}(\%)$ & & & & & 0.029 \\
\hline$<60$ & $2(100)$ & 133 (72.3) & $2(28.6)$ & $1(33.3)$ & - \\
\hline$\geq 60$ & $0(0)$ & $51(27.7)$ & $5(71.4)$ & $2(66.6)$ & - \\
\hline Sex - n (\%) & & & & & 0.303 \\
\hline Male & $0(0)$ & $91(49.5)$ & $5(71.4)$ & $2(66.6)$ & - \\
\hline Female & $2(100)$ & $93(50.5)$ & $2(28.6)$ & 1 (33.3) & - \\
\hline Smoking- n (\%) & $0(0)$ & $12(6.5)$ & $1(14.3)$ & $0(0)$ & 0.796 \\
\hline Drinking- n (\%) & $0(0)$ & $15(8.2)$ & $1(14.3)$ & $0(0)$ & 0.851 \\
\hline Food allergy- $n(\%)$ & $0(0)$ & $0(0)$ & $0(0)$ & $0(0)$ & - \\
\hline Drug allergy- n (\%) & $0(0)$ & $15(8.2)$ & $0(0)$ & $0(0)$ & 0.787 \\
\hline Surgery- n (\%) & $0(0)$ & $27(14.7)$ & $1(14.3)$ & $0(0)$ & 0.836 \\
\hline \multicolumn{6}{|c|}{ Previous coexisting disease $-\mathrm{n}(\%)$} \\
\hline Hypertension & $0(0)$ & $42(22.8)$ & $3(42.9)$ & $1(33.3)$ & 0.516 \\
\hline Diabetes & $0(0)$ & $18(9.8)$ & $2(28.6)$ & $1(33.3)$ & 0.227 \\
\hline Heart disease & $0(0)$ & $7(3.8)$ & $2(28.6)$ & $0(0)$ & 0.021 \\
\hline Pulmonary disease & $0(0)$ & $2(10.9)$ & $0(0)$ & $1(33.3)$ & $<0.001$ \\
\hline Liver disease & $0(0)$ & $10(5.4)$ & $0(0)$ & $1(33.3)$ & 0.179 \\
\hline Kidney disease & $0(0)$ & $3(1.6)$ & $0(0)$ & $0(0)$ & 0.978 \\
\hline Origin- $\mathrm{n}(\%)$ & & & & & $<0.001$ \\
\hline Inland & $0(0)$ & $166(90.2)$ & $6(85.7)$ & $3(100)$ & - \\
\hline Oversea & $2(100)$ & $18(9.8)$ & $1(14.3)$ & $0(0)$ & - \\
\hline
\end{tabular}


Table 2

Frequency of signs and symptoms according to COVID-19 phenotypes.

\begin{tabular}{|c|c|c|c|c|c|}
\hline Signs and symptoms & $\begin{array}{l}\text { Mild } \\
(n=2)\end{array}$ & $\begin{array}{l}\text { Common } \\
(n=184)\end{array}$ & $\begin{array}{l}\text { Severe } \\
(n=7)\end{array}$ & $\begin{array}{l}\text { Critical } \\
(n=3)\end{array}$ & $\begin{array}{l}\text { Total } \\
(n=196)\end{array}$ \\
\hline Fever & $0(0)$ & $155(84.2 \%)$ & $7(100 \%)$ & $3(100 \%)$ & $165(84.2 \%)$ \\
\hline Dry cough & $2(100 \%)$ & $83(45.1 \%)$ & $3(42.9 \%)$ & $1(33.3 \%)$ & $89(45.4 \%)$ \\
\hline Expectoration & $1(50 \%)$ & $30(16.3 \%)$ & $2(28.6 \%)$ & $1(33.3 \%)$ & $34(17.3 \%)$ \\
\hline Dyspnea & $0(0)$ & $7(3.8 \%)$ & $1(14.3 \%)$ & $0(0)$ & $8(4.1 \%)$ \\
\hline Headache & $0(0)$ & $11(6 \%)$ & $0(0)$ & $0(0)$ & $11(5.6 \%)$ \\
\hline Dizziness & $0(0)$ & $5(2.7 \%)$ & $0(0)$ & $0(0)$ & $5(2.6 \%)$ \\
\hline Fatigue & $0(0)$ & $7(3.8 \%)$ & $0(0)$ & $0(0)$ & $7(3.6 \%)$ \\
\hline Sore/Itchy/dry throat & $0(0)$ & $12(6.5 \%)$ & $0(0)$ & $0(0)$ & $12(6.1 \%)$ \\
\hline Nasal congestion & $0(0)$ & $7(3.8 \%)$ & $0(0)$ & $0(0)$ & $7(3.5 \%)$ \\
\hline Runny nose & $0(0)$ & $7(3.8 \%)$ & $0(0)$ & $0(0)$ & $7(3.5 \%)$ \\
\hline Diarrhea & $0(0)$ & $3(1.6 \%)$ & $0(0)$ & $0(0)$ & $3(1.5 \%)$ \\
\hline Myalgia & $0(0)$ & $5(2.7 \%)$ & $0(0)$ & $0(0)$ & $5(2.5 \%)$ \\
\hline Chilly & $0(0)$ & $8(4.3 \%)$ & $0(0)$ & $0(0)$ & $8(4.1 \%)$ \\
\hline Others symptoms & $0(0)$ & $23(12.5 \%)$ & $0(0)$ & $1(33.3 \%)$ & $24(12.2 \%)$ \\
\hline \multicolumn{6}{|l|}{ Initial therapy } \\
\hline Oxygen therapy & $0(0)$ & $56(30.4 \%)$ & $7(100 \%)$ & $3(100 \%)$ & $66(33.7 \%)$ \\
\hline Prezcobix & $0(0)$ & $14(7.6 \%)$ & $0(0)$ & $1(33.3 \%)$ & $15(7.7 \%)$ \\
\hline Arbidol & $1(50 \%)$ & $40(21.7 \%)$ & $2(28.6 \%)$ & $2(66.6 \%)$ & $45(22.9 \%)$ \\
\hline Interferon & $0(0)$ & $71(38.6 \%)$ & $2(28.6 \%)$ & $1(33.3 \%)$ & $74(37.8 \%)$ \\
\hline Lopinavir and Ritonavir Tablets & $0(0)$ & $37(20.1 \%)$ & $2(28.6 \%)$ & $1(33.3 \%)$ & $40(20.4 \%)$ \\
\hline Meropenem & $0(0)$ & $1(0.5 \%)$ & $0(0)$ & $1(33.3 \%)$ & $2(1 \%)$ \\
\hline Moxifloxacin & $0(0)$ & $15(8.1 \%)$ & $0(0)$ & $1(33.3 \%)$ & $16(8.2 \%)$ \\
\hline Omeprazole & $0(0)$ & $3(1.6 \%)$ & $0(0)$ & $2(66.6 \%)$ & $5(1.6 \%)$ \\
\hline Vitamin C & $0(0)$ & $15(8.2 \%)$ & $1(14.3 \%)$ & $1(33.3 \%)$ & $17(8.7 \%)$ \\
\hline Hydroxychloroquine & $0(0)$ & $7(3.8 \%)$ & $1(14.3 \%)$ & $0(0)$ & $8(4.1 \%)$ \\
\hline
\end{tabular}

Signs and symptoms were collected from the electronic medical records for the hospitalized patients 


\begin{tabular}{|llllll|}
\hline Signs and symptoms & $\begin{array}{l}\text { Mild } \\
(\mathbf{n = 2})\end{array}$ & $\begin{array}{l}\text { Common } \\
(\mathbf{n}=\mathbf{1 8 4})\end{array}$ & $\begin{array}{l}\text { Severe } \\
(\mathbf{n}=\mathbf{7})\end{array}$ & $\begin{array}{l}\text { Critical } \\
(\mathbf{n}=\mathbf{3})\end{array}$ & $\begin{array}{l}\text { Total } \\
(\mathbf{n}=\mathbf{1 9 6})\end{array}$ \\
\hline ShuFengJieDuJiaoNang & $0(0)$ & $10(5.4 \%)$ & $1(14.3 \%)$ & $0(0)$ & $11(5.6 \%)$ \\
\hline Others agents & $0(0)$ & $27(14.7 \%)$ & $1(14.3 \%)$ & $2(66.6 \%)$ & $30(15.3 \%)$ \\
\hline Signs and symptoms were collected from the electronic medical records for the hospitalized patients \\
\hline 3.2. AsSOCiation Of ACE2 SNPS and COVID-19
\end{tabular}

To find out further relationships between the 5 ACE2 polymorphisms and COVID-19, we used LightCycler®480 II system for TaqMan $® S N P$ Genotyping Assays and LightCycler®480SW1.5.1 software for data analysis (Figure 1). Among the results of rs2048683 ( $T>G$ ) genotyping (Fig. 1A, F), FAM fluorescent labeled probe targeted at base $\mathrm{T}$ and VIC fluorescent labeled probe targeted at base $\mathrm{G}$. The prediction analysis of rs2048683 locus of 210 normal subjects (Fig. 1A) in peripheral blood was as follows: TT genotype 0 cases, TG genotype 0 cases, GG genotype 192 cases (red dots), and 18 cases were not classified into different genotypes (blue dots). The predicted analysis of the locus of 196 patients with COVID-19 (Fig. 1F)were as follows: TT genotype 1 case (blue dots), TG genotype 22 cases (green dots), GG genotype 168 cases (red dots), and 18 cases were not classified into different genotypes (purple dots). Among the results of rs4240157 (C > T) genotyping (Fig. 1B, G), FAM fluorescent labeled probe targeted at base $\mathrm{T}$ and $\mathrm{VIC}$ fluorescent labeled probe targeted at base $\mathrm{C}$. The prediction analysis of rs4240157 locus of 210 normal subjects (Fig. 1B) in peripheral blood was as follows: CC genotype 7 cases (red dots), CT genotype 11 cases (green dots), TT genotype 181 cases (blue dots), and 11 cases were not classified into different genotypes (purple dots). The predicted analysis of the locus of 196 patients with COVID-19 (Fig. 1G) were as follows: CC genotype 7 case (red dots), CT genotype 4 cases (green dots), TT genotype 183 cases (blue dots), and 2 cases were not classified into different genotypes (purple dots). Among the results of rs4646142 (G > C) genotyping (Fig. 1C, H), FAM fluorescent labeled probe targeted at base $\mathrm{G}$ and VIC fluorescent labeled probe targeted at base $\mathrm{C}$. The prediction analysis of rs4646142 locus of 210 normal subjects (Fig. 1C) in peripheral blood was as follows: CC genotype 81 cases (red dots), GC genotype 73 cases (green dots), GG genotype 53 cases (blue dots), and 3 cases were not classified into different genotypes (purple dots).The predicted analysis of the locus of 196 patients with COVID-19 (Fig. 1H) were as follows: CC genotype 76 case (red dots), GC genotype 51 cases (green dots), GG genotype 64 cases (blue dots), and 5 cases were not classified into different genotypes (purple dots). Among the results of rs6632677 (G > C) genotyping (Fig. 1D, I), FAM fluorescent labeled probe targeted at base $\mathrm{G}$ and $\mathrm{VIC}$ fluorescent labeled probe targeted at base $\mathrm{C}$. The prediction analysis of rs6632677 locus of 210 normal subjects (Fig. 1D) in peripheral blood was as follows: CC genotype 37 cases (red dots), GC genotype 43 cases (green dots), GG genotype 122 cases (blue dots), and 3 cases were not classified into different genotypes (purple dots).The predicted analysis of the locus of 196 patients with COVID-19 (Fig. 1I) were as follows: CC genotype 12 case (red dots), GC genotype 15 cases (green dots), GG genotype 169 cases (blue dots), and 5 cases were not classified into different genotypes (purple dots). Among the results of rs2074192 (C > T) genotyping (Fig. 1E, J), FAM fluorescent labeled 
probe targeted at base $\mathrm{T}$ and $\mathrm{VIC}$ fluorescent labeled probe targeted at base $\mathrm{C}$. The prediction analysis of rs2074192 locus of 210 normal subjects (Fig. 1E) in peripheral blood was as follows: CC genotype 84 cases (red dots), CT genotype 69 cases (green dots), TT genotype 53 cases (blue dots), and 19 cases were not classified into different genotypes (purple dots). The predicted analysis of the locus of 196 patients with COVID-19 (Fig. $1 \mathrm{~J}$ ) were as follows: CC genotype 84 case (red dots), CT genotype 48 cases (green dots), TT genotype 59 cases (blue dots), and 5 cases were not classified into different genotypes (purple dots).

Next, we compared the genotype/allele frequencies between COVID-19 patients and normal controls, genotype/allele frequencies for ACE2 SNPs rs2074192, rs6632677, rs4646142, rs2048683, rs4240157 were listed (Table 3). Since genotype TT and GT of rs2024683 appeared zero in the normal group, data statistics could not be conducted, rs2024683 were eliminated. Base on Chi-square-test multiple inheritance models, the ACE2 SNP rs4240157 genotype frequency compare with control groups, without statistically significant differences. The dominant (OR $=0.24,95 \% \mathrm{Cl} ; 0.15-0.40, P<0.001$ ), recessive (OR $=3.44,95 \% \mathrm{Cl} ; 1.71-6.58, P=0.0002)$, over-dominant $(\mathrm{OR}=3.26,95 \% \mathrm{Cl} ; 1.79-6.28, P<0.001)$ model were all showed that rs 6632677 is significantly associated with the prevalence of COVID-19, and the recessive (OR $=0.56,95 \% \mathrm{Cl} ; 0.35-0.90, P=0.014)$, over-dominant ( $\mathrm{OR}=1.69,95 \% \mathrm{Cl} ; 1.08-2.62, P=0.020)$ model were showed that rs2074192 was significantly related to COVID-19. For rs4646142, there were also significant differences between GC and GG genotypes ( $\mathrm{OR}=0.58,95 \% \mathrm{Cl} ; 0.34-0.96, P=0.035)$. These results suggested that ACE2 SNPs rs4646142, rs6632677 and rs2074192 were different between normal subjects and COVID-19 patients in our population. 
Table 3

Genotype and allele distribution of ACE2 SNPS.

\begin{tabular}{|c|c|c|c|c|c|}
\hline SNP & $\begin{array}{l}\text { Genotype/Allele } \\
\text { frequency }\end{array}$ & Normal & COVID-19 & $\begin{array}{l}\text { Odd ratio }(95 \% \\
\text { Cl) }\end{array}$ & $\begin{array}{l}P \\
\text { value }\end{array}$ \\
\hline \multirow[t]{8}{*}{ rs4646142 } & GG & $53(25.6 \%)$ & $64(33.5 \%)$ & Ref & \\
\hline & GC & $73(35.3 \%)$ & $51(26.7 \%)$ & $0.58(0.34-0.96)$ & 0.035 \\
\hline & $\mathrm{CC}$ & $81(39.1 \%)$ & $76(39.8 \%)$ & $0.78(0.48-1.25)$ & 0.303 \\
\hline & G & $179(43 \%)$ & $179(47 \%)$ & $0.86(0.66-1.14)$ & 0.305 \\
\hline & $\mathrm{C}$ & $235(57 \%)$ & $203(53 \%)$ & & \\
\hline & \multicolumn{3}{|l|}{ CC vs GC+GG (dominant) } & $0.97(0.65-1.45)$ & 0.893 \\
\hline & \multicolumn{3}{|l|}{ CC+GC vs GG (recessive) } & $0.68(0.45-1.06)$ & 0.084 \\
\hline & \multicolumn{3}{|c|}{$\mathrm{CC}+\mathrm{GG}$ vs GC (over-dominant) } & $1.50(0.98-2.27)$ & 0.065 \\
\hline \multirow[t]{8}{*}{ rs20248683 } & TT & $0(0 \%)$ & $1(0.5 \%)$ & Ref & \\
\hline & GT & $0(0 \%)$ & $22(11.5 \%)$ & - & - \\
\hline & GG & $192(100 \%)$ & $168(88 \%)$ & $0(0-7.92)$ & 0.286 \\
\hline & $\mathrm{T}$ & $0(0 \%)$ & $24(6 \%)$ & $0(0-0.13)$ & $\hat{0.001}$ \\
\hline & G & $384(100 \%)$ & $358(94 \%)$ & & \\
\hline & \multicolumn{3}{|l|}{ GG vs GT+TT (dominant) } & $\mathrm{Na}(6.96-\mathrm{Na})$ & $\dot{0} .001$ \\
\hline & \multicolumn{3}{|l|}{ GG+GT vs TT (recessive) } & $0(0-8.95)$ & 0.315 \\
\hline & \multicolumn{3}{|c|}{ GT vs GG+TT (over-dominant) } & $0(0-0.14)$ & $\hat{0.001}$ \\
\hline \multirow[t]{7}{*}{ rs4240157 } & $\mathrm{CC}$ & $7(3.5 \%)$ & $7(3.6 \%)$ & Ref & \\
\hline & $\mathrm{CT}$ & $11(5.5 \%)$ & $4(2.1 \%)$ & $0.36(0.09-1.59)$ & 0.196 \\
\hline & TT & $181(91 \%)$ & $\begin{array}{l}183 \\
(94.3 \%)\end{array}$ & $1.01(0.38-2.71)$ & 0.984 \\
\hline & C & $25(6 \%)$ & $18(5 \%)$ & $1.38(0.76-2.50)$ & 0.311 \\
\hline & $\mathrm{T}$ & $373(94 \%)$ & $370(95 \%)$ & & \\
\hline & \multicolumn{3}{|l|}{ TT vs CT+CC (dominant) } & $0.60(0.27-1.28)$ & 0.201 \\
\hline & \multicolumn{3}{|l|}{ TT+CT vs CC (recessive) } & $0.97(0.36-2.61)$ & 0.961 \\
\hline
\end{tabular}

$\mathrm{P}$ value is for Chi-square test, Abbreviation: $\mathrm{OR}$, odds ratio, $\mathrm{Cl}$, Confidence Interval. 


\begin{tabular}{|c|c|c|c|c|c|}
\hline SNP & $\begin{array}{l}\text { Genotype/Allele } \\
\text { frequency }\end{array}$ & Normal & COVID-19 & $\begin{array}{l}\text { Odd ratio (95\% } \\
\text { Cl) }\end{array}$ & $\begin{array}{l}\mathrm{P} \\
\text { value }\end{array}$ \\
\hline & \multicolumn{3}{|c|}{ CT vs TT+CC (over-dominant) } & $2.78(0.95-8.06)$ & 0.073 \\
\hline \multirow[t]{8}{*}{ rs6632677 } & GG & $\begin{array}{l}122 \\
(60.4 \%)\end{array}$ & $\begin{array}{l}169 \\
(86.2 \%)\end{array}$ & Ref & \\
\hline & $\mathrm{GC}$ & $43(21.3 \%)$ & $15(7.7 \%)$ & $0.25(0.13-0.47)$ & $\dot{\delta} 001$ \\
\hline & $\mathrm{CC}$ & $37(18.3 \%)$ & $12(6.1 \%)$ & $0.23(0.12-0.46)$ & <. 001 \\
\hline & G & $287(71 \%)$ & $353(90 \%)$ & $0.27(0.18-0.40)$ & $\dot{0.001}$ \\
\hline & C & $117(29 \%)$ & $39(10 \%)$ & & \\
\hline & \multicolumn{3}{|c|}{ GG vs GC+CC (dominant) } & $0.24(0.15-0.40)$ & $\begin{array}{l}<.001 \\
0.00\end{array}$ \\
\hline & \multicolumn{3}{|c|}{ GG+GC vs CC (recessive) } & $3.44(1.71-6.58)$ & $\dot{0.001}$ \\
\hline & \multicolumn{3}{|c|}{ GC vs GG+CC (over-dominant) } & $3.26(1.79-6.28)$ & $\begin{array}{l}<.001 \\
0.00\end{array}$ \\
\hline \multirow[t]{8}{*}{ rs2074192 } & $\mathrm{CC}$ & $84(44 \%)$ & $84(44 \%)$ & Ref & \\
\hline & $\mathrm{CT}$ & $69(36.1 \%)$ & $48(25.1 \%)$ & $0.70(0.43-1.11)$ & 0.135 \\
\hline & $\mathrm{TT}$ & $38(19.9 \%)$ & $59(30.9 \%)$ & $1.55(0.95-2.60)$ & 0.089 \\
\hline & C & $237(62 \%)$ & $216(57 \%)$ & $1.26(0.94-1.67)$ & 0.122 \\
\hline & $\mathrm{T}$ & $145(38 \%)$ & $166(43 \%)$ & & \\
\hline & \multicolumn{3}{|c|}{ CC vs CT+TT (dominant) } & $1(0.67-1.50)$ & $\begin{array}{l}> \\
0.999\end{array}$ \\
\hline & \multicolumn{3}{|c|}{ CC+CT vs TT (recessive) } & $0.56(0.35-0.90)$ & 0.014 \\
\hline & \multicolumn{3}{|c|}{ CT vs CC+TT (over-dominant) } & $1.69(1.08-2.62)$ & 0.020 \\
\hline \multicolumn{6}{|c|}{$\mathrm{P}$ value is for Chi-square test, Abbreviation: OR, odds ratio, $\mathrm{Cl}$, Confidence Interval. } \\
\hline
\end{tabular}

C-reactive protein (CRP) is the main acute phase protein with a rapid and sharp increase in plasma concentration during infection and tissue damage, which can activate complement system and strengthen the phagocytosis of phagocytes. Acid glycoprotein (AGP) is the main acute response protein, which is increased during acute inflammation. Obviously, CRP and AGP play a vital protective role in the body's innate immune process. As shown in Fig. 2A.F, COVID-19 related ACE2 SNP rs4646142 was associated with increased CRP $(P<0.001)$ and AGP $(P<0.001)$, and rs6632677 may also be associated 
with increased CRP $(P=0.052)$. High-density lipoprotein $(\mathrm{HDL})$ is a plasma lipoprotein that resists atherosclerosis and is a protective factor for coronary heart disease. Apolipoprotein A (ApoA) is also an important component of plasma HDL, which can effectively remove lipids from tissues and is considered ananti-atherosclerosis factor. As shown in Fig. 2C.D. ACE2 SNP rs4646142 were correlated with the abnormality of ApoA $(P=0.003)$ and $\mathrm{HDL}(P=0.009)$. For Fig. 2B. ACE2 SNP rs 4646142 was significantly associated with prealbumin (PAB) $(P=0.044)$, and $\mathrm{PAB}$ is used as a sensitive nutritional protein indicator. For Fig. 2E. ACE2 SNP rs6632677 was further associated with HPT ( $P=0.027)$, and rs4646142 may also be associated with HPT although there is no significant difference $(P=0.083)$. Dramatically, ACE2 SNP rs2074192 was not correlated with any clinical indicators.

\section{Discussion}

Notably different responses to SARS-CoV-2 infection among different populations raise the possibility that different SNPs profile might be responsible for the risk of acute inflammation, cardiovascular, hypertension, diabetes, and stroke in COVID-19patients. In the present study, we identified in our population ACE2 SNPs rs4646142, rs6632677 and rs2074192, are associated with COVID-19 following SARS-CoV-2 infection. As far as we know, this is the first genetic study that has shown rs4646142, rs6632677 and rs2074192 may increase the odds of COVID-19 infection with previous coexisting disease in Chinese. Recent reports in Caucasian individuals determined the ACE2 rs2285666 variant was related to hypertension in elderly population(24). Taken together, the findings indicate a prominent role of ACE2 polymorphisms in the pathogenesis of COVID-19.

ACE2 is a transmembrane protein and the main entry point for some coronaviruses into cells, including SARS-CoV and SARS-CoV-2(25). ACE2 may be related to a higher number of membrane-bound viral binding sites, which causing the vulnerability of carriers to infection. After the receptor binding domain (RBD) in the S1 domain of SARS-CoV and SARS-CoV-2 viral spike proteins bind to the extracellular region of ACE2, and spikes may be cleaved by TMPRSS2 on the cell surface, promoting the fusion of the outer membrane of the virus and the host cell membrane to allow the virus to enter the cytoplasm (26). Previous studies have reported that among patients with COVID-19 and coexisting hypertension, the mortality was lower through taking ACE inhibitors or angiotensin II receptor blockers (ARBs), when compared with COVID- 19 patients with hypertension not taking such drugs (27). One study put forward a hypothesis on the role of Renin-Angiotensin-System (RAS) pathway genes including ACE2 (rs2285666, rs1978124, rs714205) in COVID-19 prognosis: inherited genetic predispositions can forecast the degree of severity of COVID-19 (28). Besides, the rs2285666 allele (T or A) was significantly positively correlated with lower infection and mortality among Indian population (29).

As can be seen from the baseline clinical characteristics (Table 1), the pathological degree of the COVID19 is associated with previous coexisting disease, especially hypertension and diabetes. In addition, based on the literature search (https://pubmed.ncbi.nlm.nih.gov/), ACE2 SNPs are mainly related to human cardiovascular, hypertension, dyslipidemia, left myocardial hypertrophy, diabetes, stroke, retinopathy, etc (Table S1). Next, through the sequence of UCSC hg19 (exome) and the reference gene 
Homo sapiens angiotensin I converting enzyme 2 (ACE2, NG_012575.1:6110-46037) on chromosome X, 5 ACE2 SNPs (rs4646142 G > A or C, rs2048683 T > A or G, rs4240157 C > G or T, rs6632677 G > C, rs2074192 C > T) known to be associated with human cardiovascular diseases and diabetes were checked. Notably, research showed that SARS-CoV-2 is most closely associated with bat coronaviruses, $100 \%$ amino acid resemblance to bat SL-CoVZC45 in the E proteins and nsp7. That is, bats are a possible host of SARS-CoV-2 virus (30). Interestingly, rs4646142, rs2048683, rs4240157, rs6632677 and rs2074192 were completely consistent with bats ACE2 SNPs, while the SNPs in snakes were significantly different (Fig. S1), suggesting that these SNPs may be associated with the infection of SARS-CoV-2. We hypothesized that ACE2 variants rs2074192 and rs6632677 could modified the disease outcome.

In this study, we investigated possible associations between ACE2 polymorphisms and COVID-19 in our collected population. we found that ACE2 SNPs rs4646142, rs6632677, rs2074192 might correlated with the susceptibility of COVID-19 related cardiovascular risk and acute inflammatory infection. So why exactly do these SNPs impact susceptibility to COVID-19? We found that rs4646142, rs6632677 and rs 2074192 were located in the intron region and could not encode amino acids. This may be related to the adjustment mode of the intron sequence. For instance, existing research shows that NS1 protein-RNA interactome, and NS1 primarily binds intronic sequences as a multifunctional virulence factor of the influenza a virus, thereby inhibiting cellular processes to accelerate viral gene expression (31). Human transformer 2 alpha homolog (huTRA2A) can inhibit mRNA splicing by binding to the intron silencer motif in the NS mRNA of the PR8/H1N1 virus (32). Some SNPs in tonicity-responsive enhancer binding protein (TonEBP) can impact transcription process as cis-expressed quantitative trait loci. Meanwhile, these SNPs are associated with increased risk of T2D, inflammation, hypertension, indicating that changes in TONEBP expression may be related to these phenotypes (33). In other words, these SNPs may be able to impact SARS-Cov2 infection process by binding to regulatory proteins or RNAs.

\section{Conclusions}

In conclusion, our study showed that the pathological degree of the COVID-19 may be associated with hypertension and diabetes. And, ACE2 gene variants were associated with the degree of severity in COVID-19 patients. Furthermore, ACE2 SNPs rs4646142 and rs6632677 may be an optimal genetic susceptibility marker for COVID-19 related cardiovascular complications, by evaluated the correlations between clinical indicators and SNPs. All in all, our findings supported that ACE2 SNPs rs4646142 and rs6632677 correlated with COVID-19 related cardiovascular risk, and it should be evaluated in large-scale studies.

\section{Abbreviations}

ACE2

Angiotensin-converting enzyme 2

COVID-19

Coronavirus disease 2019 
SNP

single-nucleotide polymorphism

hs-CRP

high-sensitive C-reactive protein

$\mathrm{PAB}$

prealbumin

APOA

apolipoprotein A

HDL

high-density lipoprotein

AGP

acid glycoprotein

HPT

haptoglobin

SARS-CoV-2

Severe acute respiratory syndrome coronavirus 2

PPI

protein-protein interaction

gDNA

Genomic DNA

RBD

receptor binding domain

ARBs

angiotensin II receptor blockers

RAS

Renin-Angiotensin-System

hUTRA2A

Human transformer 2 alpha homolog

TonEBP

tonicity-responsive enhancer binding protein

T2D

Type 2 diabetes mellitus.

\section{Declarations}

\section{Ethics approval and consent to participate}

All procedures were reviewed and approved by the Ethics Committee of Shanghai Public Health Clinical Center, Fudan University, and have obtained written informed consents from all subjects. 
All authors read, contributed to, and consent for publication.

\section{Competing interests}

The authors declare no conflicts of interests.

\section{Funding}

This research was supported by a grant from Science and Technology Commission of Shanghai (20Y11900700, 20411950200 and 20Z11900900), a grant from Special Research Fund of Youan Medical Alliance for the Liver and Infectious Diseases (LM202020), a grant (2018ZX10302103-003) from the National Special Research Program of China for Important Infectious Diseases and a grant from the National Natural Science Foundation of China (81672383).

\section{Authors' contributions}

All authors read, contributed to, and approved the final manuscript.

\section{Acknowledgments}

Thanks to Shanghai Public Health Clinical Center for providing samples.

\section{Availability of data and material}

The datasets used or analysed during the current study are available from the corresponding author on reasonable request.

\section{References}

1. COVID-19 Dashboard by the Center for Systems Science and Engineering (CSSE) at Johns Hopkins University (JHU) [Internet]. Johns Hopkins University. 2021-05-29.

2. Islam MA, Kundu S, Alam SS, Hossan T, Kamal MA, Hassan R. Prevalence and characteristics of fever in adult and paediatric patients with coronavirus disease 2019 (COVID-19): A systematic review and meta-analysis of 17515 patients. PLoS One. 2021;16(4):e0249788.

3. Islam MA, Alam SS, Kundu S, Hossan T, Kamal MA, Cavestro C. Prevalence of Headache in Patients With Coronavirus Disease 2019 (COVID-19): A Systematic Review and Meta-Analysis of 14,275 Patients. Front Neurol. 2020;11:562634.

4. Saniasiaya J, Islam MA, Abdullah B. Prevalence and Characteristics of Taste Disorders in Cases of COVID-19: A Meta-analysis of 29,349 Patients. Otolaryngol Head Neck Surg. 2020:194599820981018.

5. Rivett L, Sridhar S, Sparkes D, Routledge M, Jones NK, Forrest S, et al. Screening of healthcare workers for SARS-CoV-2 highlights the role of asymptomatic carriage in COVID-19 transmission. Elife. 2020;9. 
6. Wang D, Hu B, Hu C, Zhu F, Liu X, Zhang J, et al. Clinical Characteristics of 138 Hospitalized Patients With 2019 Novel Coronavirus-Infected Pneumonia in Wuhan, China. JAMA. 2020;323(11):1061-9.

7. Clerkin KJ, Fried JA, Raikhelkar J, Sayer G, Griffin JM, Masoumi A, et al. COVID-19 and Cardiovascular Disease. Circulation. 2020;141(20):1648-55.

8. Hou Y, Zhao J, Martin W, Kallianpur A, Chung MK, Jehi L, et al. New insights into genetic susceptibility of COVID-19: an ACE2 and TMPRSS2 polymorphism analysis. BMC Med. 2020;18(1):216.

9. Jiang S, Li Q, Li C, Liu S, He X, Wang T, et al. Mathematical models for devising the optimal SARSCoV-2 strategy for eradication in China, South Korea, and Italy. J Transl Med. 2020;18(1):345.

10. Shanshan Liu QL, Xuntao Chu, Minxia Zeng, Mingbin Liu,Xiaomeng He,Heng Zou, Jianghua Zheng, Christopher Corpe, Xiaoyan Zhang, Jianqing Xu and Jin Wang. Monitoring Coronavirus Disease 2019: A Review of Available Diagnostic Tools. frontiers in Public Health. 2021; Volume 9.

11. Zhang X, Tan Y, Ling Y, Lu G, Liu F, Yi Z, et al. Viral and host factors related to the clinical outcome of COVID-19. Nature. 2020;583(7816):437-40.

12. Burrell LM, Harrap SB, Velkoska E, Patel SK. The ACE2 gene: its potential as a functional candidate for cardiovascular disease. Clin Sci (Lond). 2013;124(2):65-76.

13. Liu C, Li Y, Guan T, Lai Y, Shen Y, Zeyaweiding A, et al. ACE2 polymorphisms associated with cardiovascular risk in Uygurs with type 2 diabetes mellitus. Cardiovasc Diabetol. 2018;17(1):127.

14. Luo Y, Liu C, Guan T, Li Y, Lai Y, Li F, et al. Association of ACE2 genetic polymorphisms with hypertension-related target organ damages in south Xinjiang. Hypertens Res. 2019;42(5):681-9.

15. Dong M, Zhang J, Ma X, Tan J, Chen L, Liu S, et al. ACE2, TMPRSS2 distribution and extrapulmonary organ injury in patients with COVID-19. Biomed Pharmacother. 2020;131:110678.

16. Hoffmann M, Kleine-Weber H, Schroeder S, Kruger N, Herrler T, Erichsen S, et al. SARS-CoV-2 Cell Entry Depends on ACE2 and TMPRSS2 and Is Blocked by a Clinically Proven Protease Inhibitor. Cell. 2020;181(2):271-80 e8.

17. Calcagnile M, Forgez P, Iannelli A, Bucci C, Alifano M, Alifano P. Molecular docking simulation reveals ACE2 polymorphisms that may increase the affinity of ACE2 with the SARS-CoV-2 Spike protein. Biochimie. 2021;180:143-8.

18. Paniri A, Hosseini MM, Moballegh-Eslam M, Akhavan-Niaki H. Comprehensive in silico identification of impacts of ACE2 SNPs on COVID-19 susceptibility in different populations. Gene Rep. 2021;22:100979.

19. Yin C. Genotyping coronavirus SARS-CoV-2: methods and implications. Genomics. 2020;112(5):3588-96.

20. Lippi G, Lavie CJ, Henry BM, Sanchis-Gomar F. Do genetic polymorphisms in angiotensin converting enzyme 2 (ACE2) gene play a role in coronavirus disease 2019 (COVID-19)? Clin Chem Lab Med. 2020;58(9):1415-22. 
21. Hamet P, Pausova Z, Attaoua R, Hishmih C, Haloui M, Shin J, et al. SARS-CoV-2 Receptor ACE2 Gene Is Associated with Hypertension and Severity of COVID 19: Interaction with Sex, Obesity, and Smoking. Am J Hypertens. 2021;34(4):367-76.

22. Lanjanian H, Moazzam-Jazi M, Hedayati M, Akbarzadeh M, Guity K, Sedaghati-Khayat B, et al. SARSCoV-2 infection susceptibility influenced by ACE2 genetic polymorphisms: insights from Tehran Cardio-Metabolic Genetic Study. Sci Rep. 2021;11(1):1529.

23. Yu Q, Nie SP, Wang JQ, Huang DF, Li WJ, Xie MY. Toll-like receptor 4 mediates the antitumor host response induced by Ganoderma atrum polysaccharide. J Agric Food Chem. 2015;63(2):517-25.

24. Gomez J, Albaiceta GM, Garcia-Clemente M, Lopez-Larrea C, Amado-Rodriguez L, Lopez-Alonso I, et al. Angiotensin-converting enzymes (ACE, ACE2) gene variants and COVID-19 outcome. Gene. 2020;762:145102.

25. Li F. Receptor recognition and cross-species infections of SARS coronavirus. Antiviral Res. 2013;100(1):246-54.

26. Strabelli TMV, Uip DE. COVID-19 and the Heart. Arq Bras Cardiol. 2020;114(4):598-600.

27. Zhang P, Zhu L, Cai J, Lei F, Qin JJ, Xie J, et al. Association of Inpatient Use of AngiotensinConverting Enzyme Inhibitors and Angiotensin II Receptor Blockers With Mortality Among Patients With Hypertension Hospitalized With COVID-19. Circ Res. 2020;126(12):1671-81.

28. Gemmati D, Tisato V. Genetic Hypothesis and Pharmacogenetics Side of Renin-Angiotensin-System in COVID-19. Genes (Basel). 2020;11(9).

29. Srivastava A, Bandopadhyay A, Das D, Pandey RK, Singh V, Khanam N, et al. Genetic Association of ACE2 rs2285666 Polymorphism With COVID-19 Spatial Distribution in India. Front Genet. 2020;11:564741.

30. Wu F, Zhao S, Yu B, Chen YM, Wang W, Song ZG, et al. A new coronavirus associated with human respiratory disease in China. Nature. 2020;579(7798):265-9.

31. Zhang L, Wang J, Munoz-Moreno R, Kim M, Sakthivel R, Mo W, et al. Influenza Virus NS1 Protein-RNA Interactome Reveals Intron Targeting. J Virol. 2018;92(24).

32. Zhu Y, Wang R, Yu L, Sun H, Tian S, Li P, et al. Human TRA2A determines influenza A virus host adaptation by regulating viral mRNA splicing. Sci Adv. 2020;6(25):eaaz5764.

33. Choi SY, Lee-Kwon W, Kwon HM. The evolving role of TonEBP as an immunometabolic stress protein. Nat Rev Nephrol. 2020;16(6):352-64.

\section{Figures}



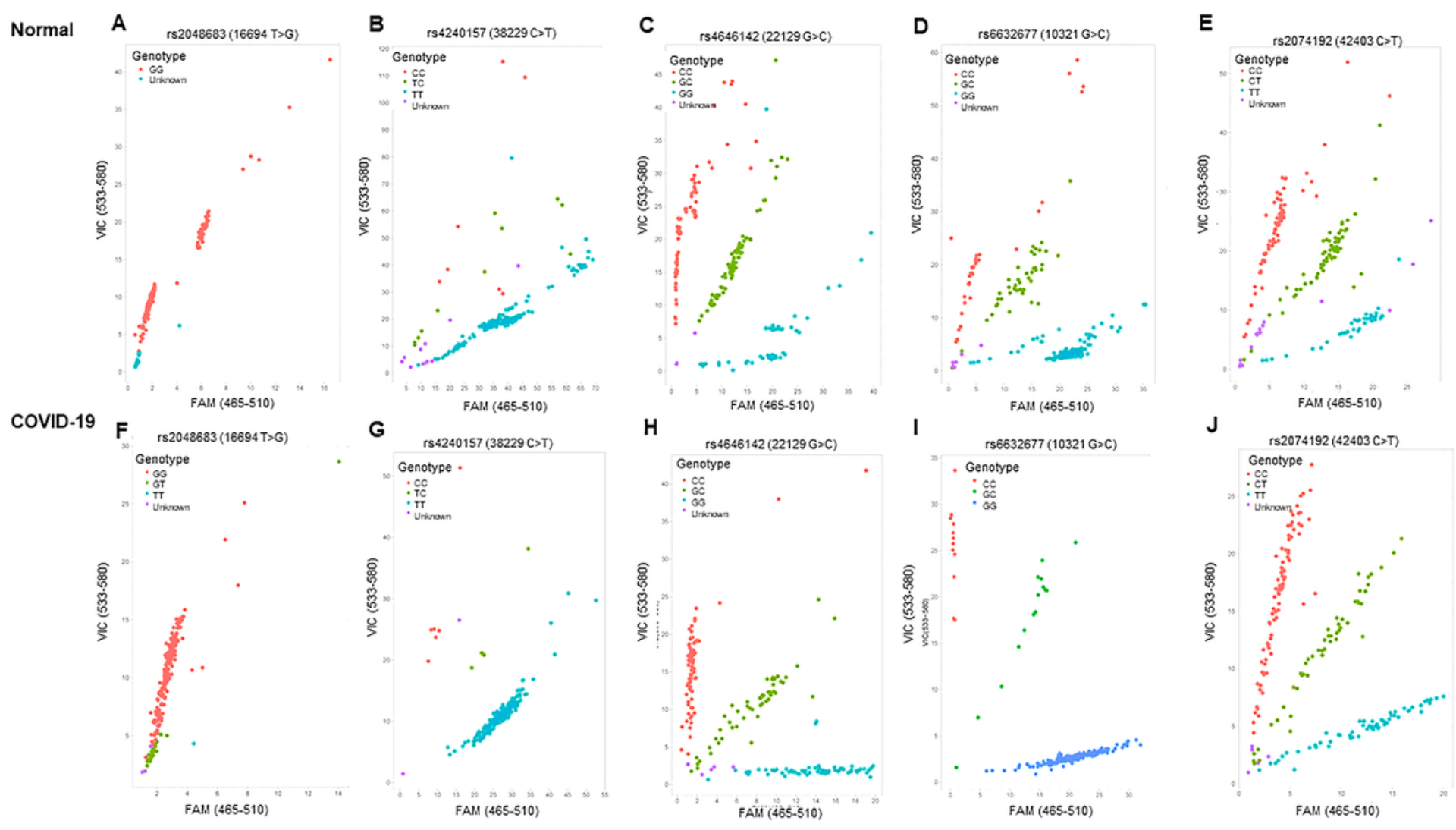

\section{Figure 1}

Fluorescence scatter diagrams of the ACE2 SNPs in normal controls (A-E) and COVID-19 patients (F-J). A, F) rs2048683; B, G) rs4240157; C, H) rs4646142; D, I) rs6632677; E, J) rs2074192. 


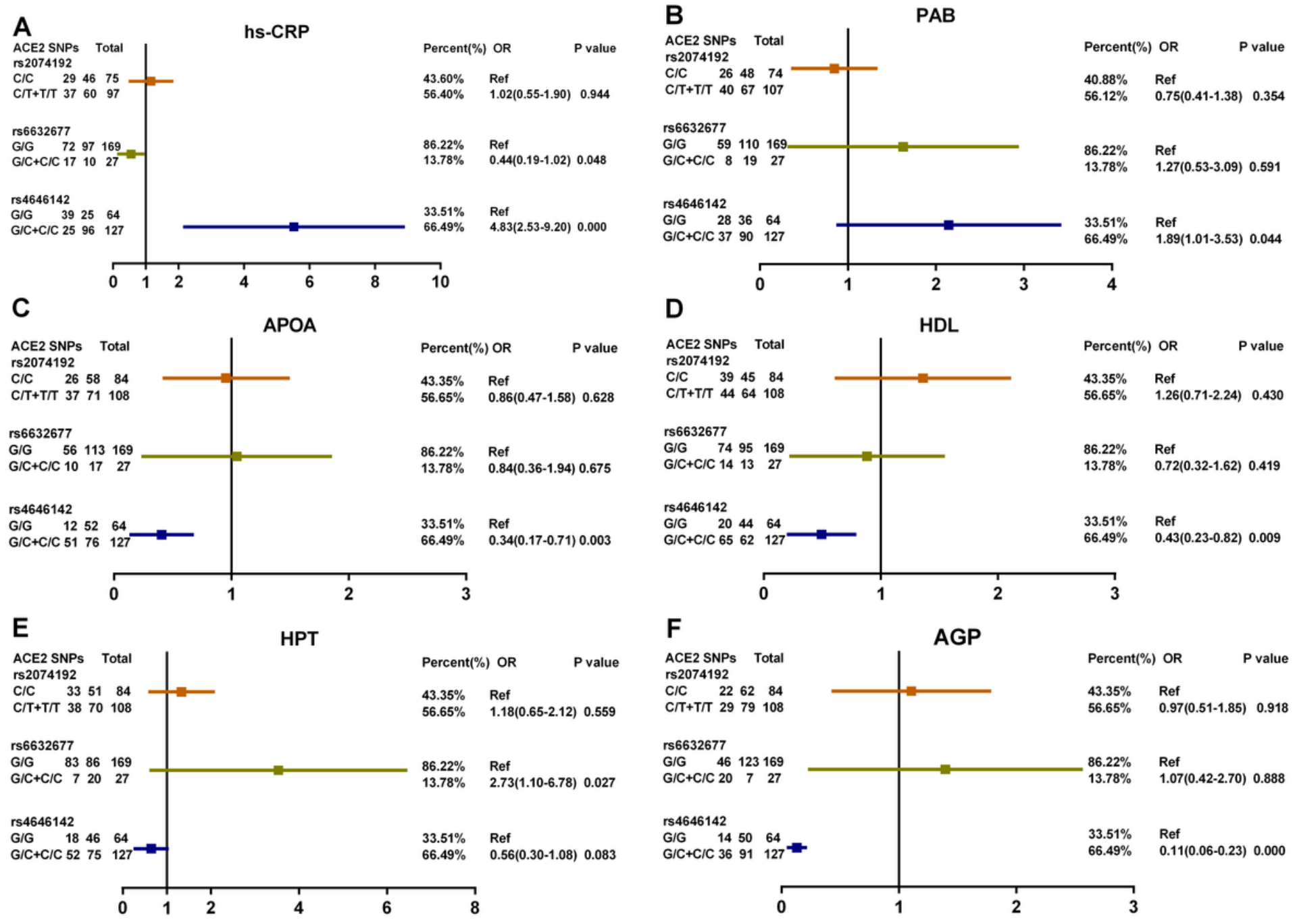

Figure 2

Associated of diseases risk related ACE2 SNPs with clinical biochemical criterion in study participants. A) A high-sensitive C-reaction (hs-CRP); B) Prealbumin (PAB); C) Apolipoprotein A (APOA); D) High-density lipoprotein (HDL); E) Haptoglobin (HPT); F) Acid glycoprotein (AGP).

\section{Supplementary Files}

This is a list of supplementary files associated with this preprint. Click to download.

- SupplementalTablesandFigures.docx 